\title{
Experimental validation of an inductive probe for narrow gap measurement based on numerical modelling
}

\author{
Edvard Svenman ${ }^{\mathrm{a}, \mathrm{b}, *}$, Anna-Karin Christiansson ${ }^{\mathrm{b}}$, Anna Runnemalm ${ }^{\mathrm{b}, \mathrm{c}}$ \\ ${ }^{a}$ GKN Aerospace Engine Systems, SE-461 81 Trollhättan, Sweden \\ ${ }^{\mathrm{b}}$ Engineering Science, University West, SE-461 86 Trollhättan, Sweden \\ ${ }^{\mathrm{c}}$ Engineering Science, University of Skövde, SE-541 28 Skövde, Sweden
}

\section{A R T I C L E I N F O}

\section{Article history:}

Received 19 January 2019

Received in revised form 17 May 2019

Accepted 15 June 2019

Available online 20 June 2019

\section{Keywords:}

Inductive sensor

Zero-gap

Measurement

Welding

\begin{abstract}
A B S T R A C T
Experimental validation of numeric results for an inductive probe shows that narrow gaps between two plates can be measured with accuracy suitable for laser beam welding. A two-coil inductive probe for measurement of the gap was built based on finite element modelling results. The individual coils were calibrated using a complex response method, and results from the physical coils closely match the numerical results regarding distance to gap and lift-off above the plate. The measurement of a realistic gap shows results that can be used in industrial applications for position, plate height and height alignment.
\end{abstract}

(c) 2019 The Authors. Published by Elsevier Ltd. This is an open access article under the CC BY-NC-ND license (http://creativecommons.org/licenses/by-nc-nd/4.0/).

\section{Introduction}

In precision welding, such as laser beam welding, the work pieces must be precisely positioned and aligned for the laser beam to be directed towards the actual gap between two plates in a butt joint configuration. Position inaccuracy can cause incomplete joining [1]. Commercial seam trackers [2] measure the weld gap using for example inductive sensors [3,4], structured light triangulating sensors [5-7] or image processing [8-10]. While optical sensing methods depend on the surface appearance and lighting conditions, inductive methods induce sensing currents into the material itself [11] and interpret the complex impedance response. Inductive sensors can respond to even very narrow gaps that interrupt these currents.

An improved method for inductive gap tracking, that can measure not only narrow gaps but also gap width and alignment, and has higher accuracy than other inductive systems, was previously proposed [3]. This system consists of two coils in a probe configuration, one coil on each side of the gap between two metal plates, see Fig. 1. This gap is the target for the sensor system. In this work commercial high sensitivity eddy current instruments are used. Driven by a high frequency alternating current, each coil changes impedance in response to both the distance to the gap and the

\footnotetext{
* Corresponding author at: GKN Aerospace Engine Systems, SE-461 81 Trollhättan, Sweden.

E-mail address: edvard.svenman@gknaerospace.com (E. Svenman).
}

height above the plate due to the varying inductive coupling between the coil and the plate. These impedance measurements are non-linear and affected by gap geometry, that is the width of the gap and the alignment of the plates' top surfaces. A calibration map is made from responses from a nominal zero-width gap for each coil. The response from each coil is then used with the respective calibration map to calculate the coil lift-off above the plate and distance to the gap. Combining the results from both coils gives a first estimate of the position $\mathrm{p}$, and height $\mathrm{h}$ of the probe, but also of the gap width w and alignment a of the plates, see Fig. 2 for definitions. These are used with an additional compensation model calculated from measurement of gaps with known width and alignment, to find corrections to improve the first estimate [12].

The instruments can be adapted to different coils, for example by using a balanced bridged configuration and by adjusting sensitivity [11]. This way the range of the instrument's amplifier can be used better. The coil response to a reference signal is split into reactive and resistive components, for example using phase sensitive demodulators, to interpret the coil response to a certain gap geometry.

When setting up experiments with inductive gap sensors, the type of coil and instrumentation must be selected to be sensitive to the desired geometry. Modern numerical signal processing makes it easy to linearize signals, but signal quality is still affected by noise and interference. Linear signals allow better use of the measurement range of amplifiers and AD-converters. Sensitivity 


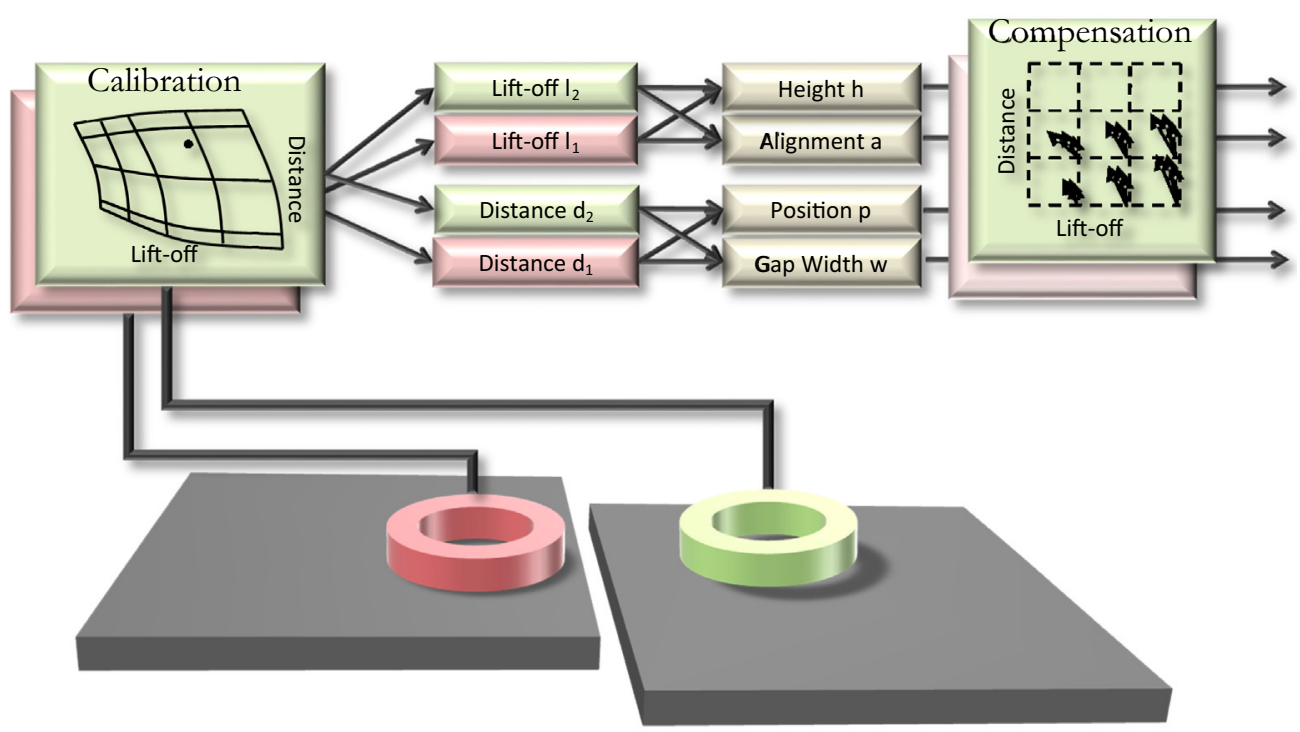

Fig. 1. Principle of complex inductive gap measurement, indicating coil 1 to the left and coil 2 to the right, their calibration functions and the operations to calculate the parameters of the gap.

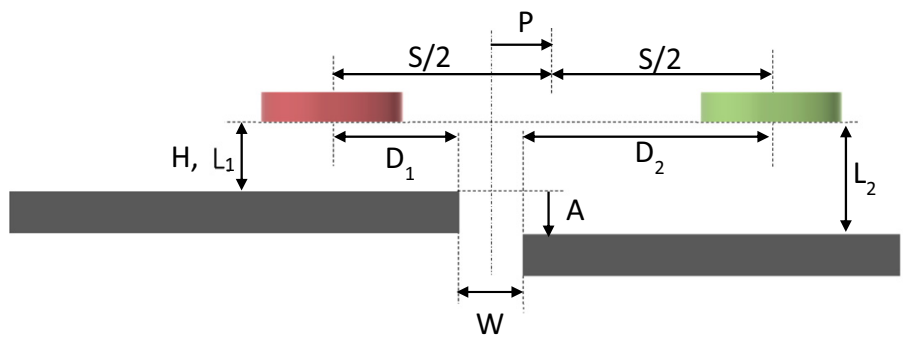

\author{
Separation: $\mathrm{S}$ \\ Distance: $D_{1}, D_{2}$ \\ Lift-off: $L_{1}, L_{2}$ \\ Position: $P=1 / 2\left(D_{2}-D_{1}\right)$ \\ Height: $H=M i n\left(L_{1}, L_{2}\right)$ \\ Width: $W=S-\left(D_{1}+D_{2}\right)$ \\ Alignment: $A=L_{2}-L_{1}$
}

Fig. 2. Definitions and nomenclature of the two coils and the gap.

is also directly related to the working range of a sensor, limited by noise, interference and resolution.

This paper presents a validation of the numerical study in [13], where the results are used to select a coil configuration for the probe in the proposed gap measurement method, Fig. 1. It also presents dual-coil measurement results from a real gap with changes in gap width and alignment, and discusses sources of uncertainty.

\section{Experimental setup}

The experiments were set up using previous experience and the modelling results $[3,12,13]$ to allow comparison of results from the numerical model, and to verify the behaviour of the physical probe that was built. The parameters of the probe and of the gap are given in Fig. 2. Upper case letters are used for reference values that are derived from for example the traverse system (see Section 2.3), while lower case letters are used for values measured by the inductive coils and probe under study. Plates and coils are numbered 1 and 2 according to side.

\subsection{Coil design and probe construction}

In the numerical study, two different coil lengths and three different coil orientations were studied [13]. It was found that longer coils had a more linear response to lift-off and distance to gap than shorter. From the three orientations, see Fig. 3, Perp was not found suitable because of a complicated response to distance D. Here, the
Norm orientation was selected because of its higher linearity and overall stronger response, both before and after normalization. The normalised sensitivity results are repeated in Fig. 4 for convenience.

Based on the results in [10] four coils were constructed by winding approximately 47 turns of $0.08 \mathrm{~mm}$ outer diameter lacquered copper wire on $2 \mathrm{~mm}$ diameter polypropylene cores, see Fig. 5(a). The length of the windings was approximately $4 \mathrm{~mm}$. The protrusion of the core, and therefore the minimum distance between the winding and the plate, was approximately $0.5 \mathrm{~mm}$. These four coils were used in pairs on each side of the gap, where one of the coils provides measurements of the metal plate, and the other coil is positioned away from the plates and is used to compensate response from temperature changes, see Fig. 5(b). Using the smallest setting supported by the probe, the two measurement coils were placed $4.1 \mathrm{~mm}$ apart.

To compare the experimental results to the numerical, a model corresponding to the coils was used to simulate the response in the same way as in the numerical investigation [13].

\subsection{Probe calibration}

Two plates of Alloy 718, $6.8 \mathrm{~mm}$ thick, were mounted in a butt joint configuration with two machined surfaces well aligned and in contact. The probe was mounted on an accurate traverse system (described in section 2.3) to allow measurement in a working range of different positions and heights around the gap. The centre 
a

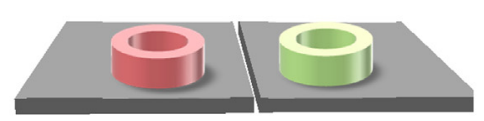

b

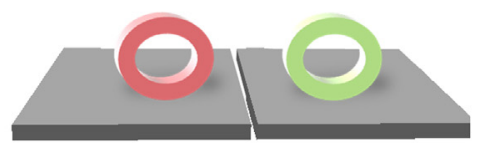

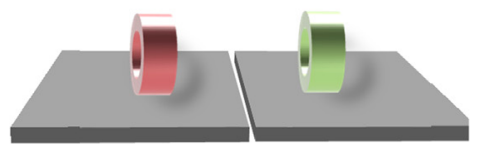

Fig. 3. Coil orientations, (a) Norm, (b) Para and (c) Perp.
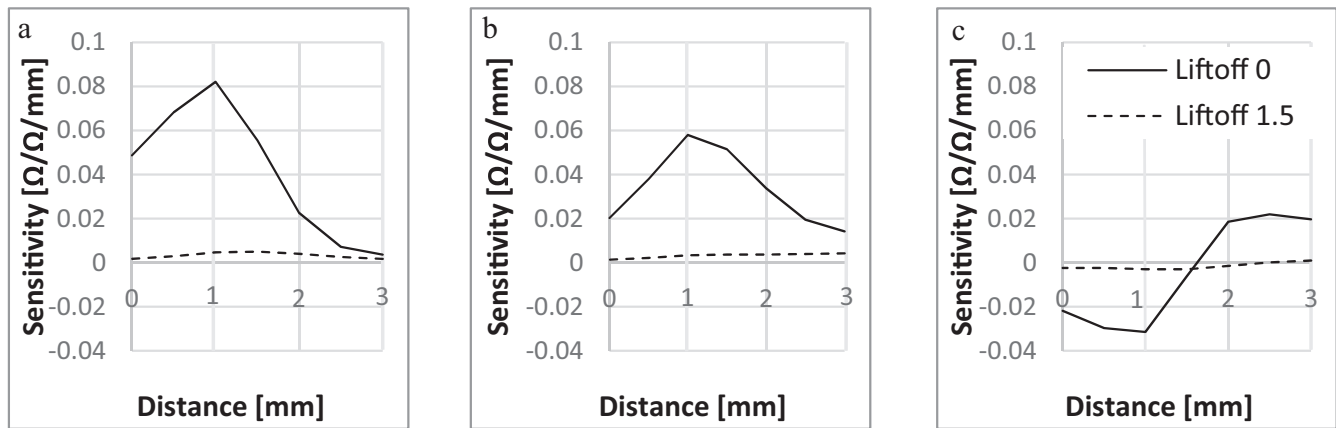

Fig. 4. Normalised sensitivity to distance for long coils (a) Norm, (b) Para (c) Perp. Values are given at lift-off $0 \mathrm{~mm}$ and $1.5 \mathrm{~mm}$. The legend in (c) applies to figures (a)-(c).

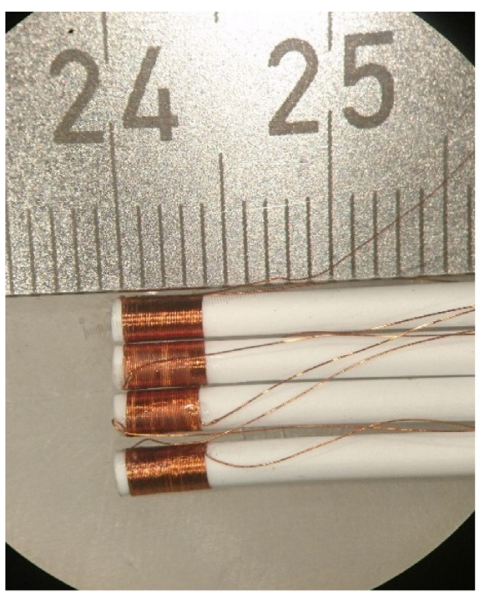

b

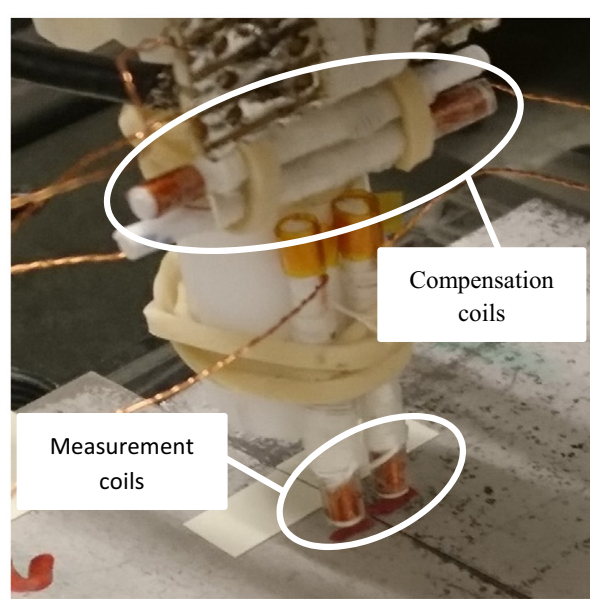

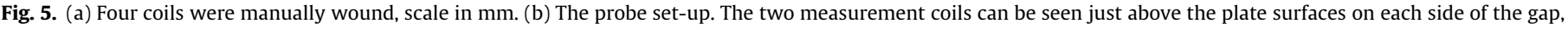
and the two compensating coils in opposite directions in the top of the picture.

position of the probe was determined by monitoring the instruments' output looking for the peak value, and visually by traversing each coil to the gap centre and monitoring the position.

Two commercial eddy current instruments, one Rohmann Elotest B1 (coil 1) and one B300 (coil 2), were used to measure the respective coil responses. All signals were recorded as voltage signals synchronised to the traverse movement, and calculations for calibration and compensation were made after the experiment. Following the results on cross sensitivity between the two coils from the numerical investigation [13], a small difference in frequency was used to let the instrument suppress response generated by the magnetic fields from the neighbouring coil and induced currents. Coil 1 was operated at $3.1 \mathrm{MHz}$, and coil 2 at 3.2 $\mathrm{MHz}$.

To assure consistent results, the instruments were zeroed before each sequence of measurements, with the coils placed in contact with the plate far from any edge. Instrument readings from scanning across a nominal zero-width gap were used to construct a calibration in the form of interpolation maps. A few additional measurements were then made on gaps adjusted to known width and alignment to calibrate the model-based compensation. These procedures are described in detail in a previous publication [12].

\subsection{Traverse and plate setup}

To verify the method, a number of measurements were made on two plates, arranged to form a $100 \mathrm{~mm}$ long gap to be measured. The edges forming the gap were machined straight. All recordings along the gap were made by an accurate traverse system at $5 \mathrm{~mm} /$ $\mathrm{s}$, and at a sample rate of $10 \mathrm{~Hz}$. The plates were roughly aligned with the traverse system. For reference, the changes in the plate 1 edge position, and in the height of both plates relative the traverse system were measured using a Micro Epsilon model 281025 ScanControl triangulating laser scanner as presented in Figs. 6 and 7. To introduce a decreasing gap width, a wire of known diameter, $0.25 \mathrm{~mm}$, was used as a distance between the two plates at the start of the measured distance, and at the end the plates were in contact. The change in gap width was derived from the wire diameter and the location along the gap. The change in position of the gap centre was derived from the direction of the plate 1 edge position. Linear polynomials were used to represent the known position $\mathrm{P}$ and width $\mathrm{W}$, see Fig. 6 which also shows the raw data and its trend-line. The measurement of the edge shows some of the problems with noise and systematic deviations in this optical measurement. With outliers deviating more than $0.4 \mathrm{~mm}$ excluded, the 


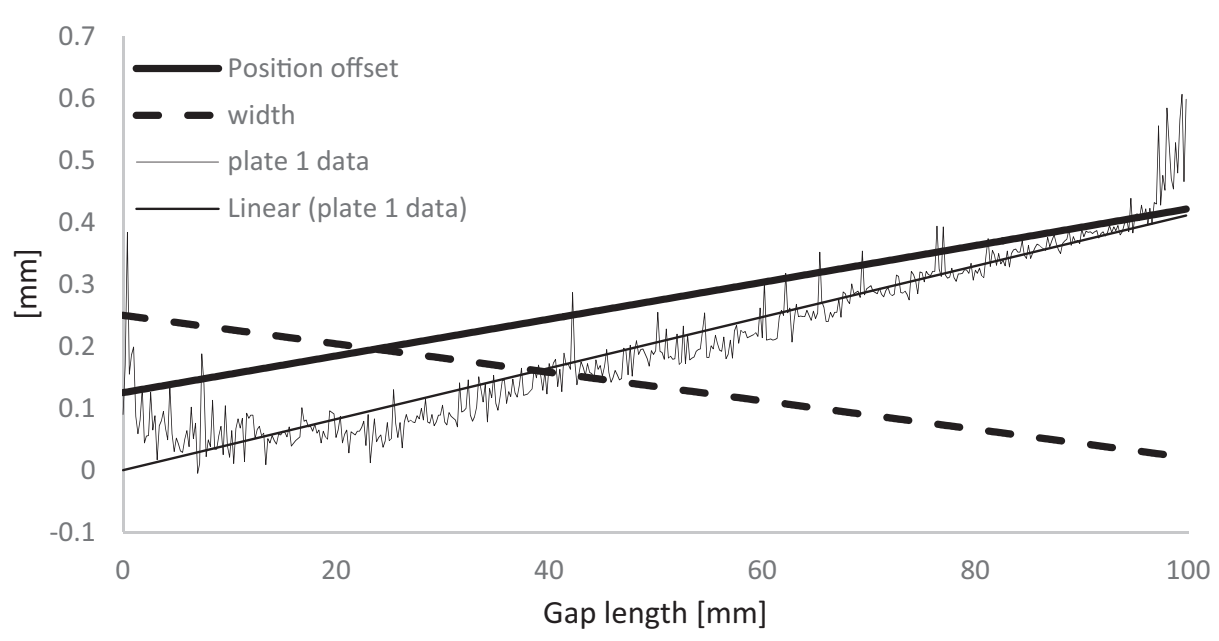

Fig. 6. Gap position offset and width, with laser scanner data and trend line for plate 1 edge.

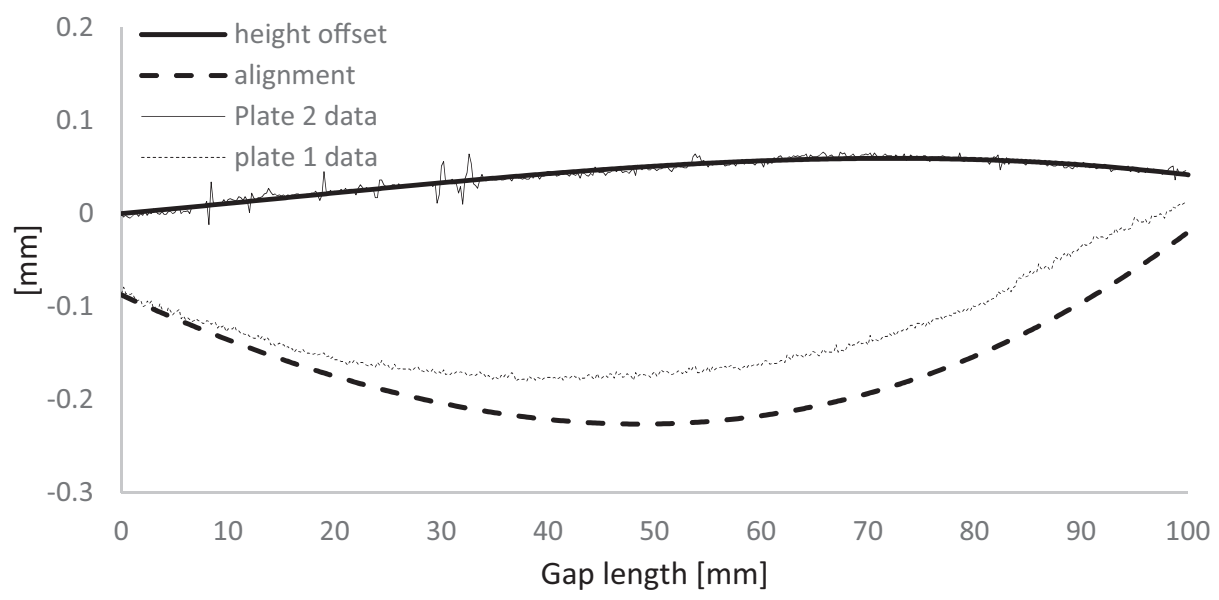

Fig. 7. Height offset and alignment, with laser scanner data for plate 1 and 2.

standard error of regression accounts for $0.09 \mathrm{~mm}$ uncertainty in position. This is accepted as a conservative estimate.

Third order polynomials were used to represent the known height $\mathrm{H}$ and alignment $\mathrm{A}$, see Fig. 7 which also shows the raw data from the laser scanner of both plates. These measurements show standard error of regression contributing less than $0.01 \mathrm{~mm}$ to uncertainty. The figures present the offset from the position measured at the start of the gap length on plate 1 for position, and on plate 2 for height.

The inductive probe measurements of the gap were performed along the gap at several positions (i.e. distance to the gap) and heights above the plate, see Fig. 8 . Positions from -1 to $+1 \mathrm{~mm}$ in steps of $0.2 \mathrm{~mm}$, and heights of 0.2 to $1.0 \mathrm{~mm}$ in steps of $0.2 \mathrm{~mm}$ were traversed. In all 55 measurements were made along $100 \mathrm{~mm}$ of the gap. The probe was visually centred over the gap using the two measurement coil centres as reference before starting the scan along the gap in this pattern.

\subsection{Uncertainty}

There are several sources of uncertainty in the experiment. Electrical noise corresponds to a distance D of less than $0.01 \mathrm{~mm}$ in the most sensitive range of the coil, but up to $0.2 \mathrm{~mm}$ at a distance of $4 \mathrm{~mm}$ from the gap. The noise contribution to lift-off is smaller than $0.01 \mathrm{~mm}$ in the entire range. The traverse system is accurate to within $0.04 \mathrm{~mm}$, the laser line measurement of position

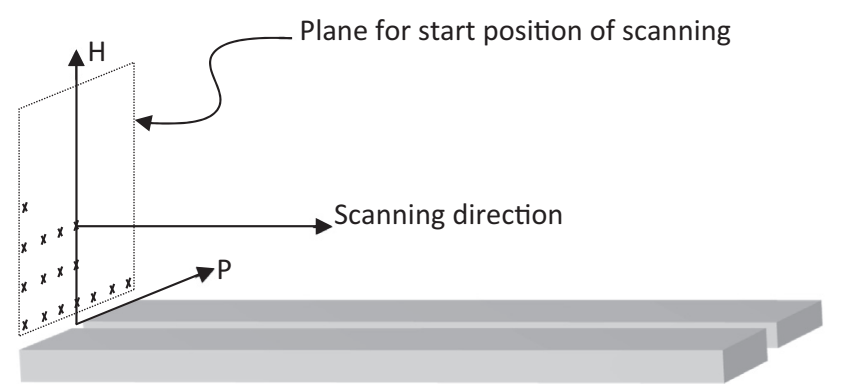

Fig. 8. Representation of the two plates and the scan pattern traversed by the probe above the gap between the plates. The nominal working range of the probe is marked centred above the start of the gap, and the directions of probe position, $\mathrm{P}$, relative the gap and height, $\mathrm{H}$, above the plates are indicated.

within $0.09 \mathrm{~mm}$ and of height within $0.01 \mathrm{~mm}$. Manual procedures such as probe centering is estimated to be accurate within $0.1 \mathrm{~mm}$, and zero height adjustment within $0.02 \mathrm{~mm}$. Error in lift-off due to error in probe angle relative the plates is estimated to be smaller than $0.04 \mathrm{~mm}$. Instrument drift on the order of $20 \mathrm{mV}$ has been observed after measurement runs, corresponding to a distance error of $0.05 \mathrm{~mm}$ in the most sensitive range, but close to $1 \mathrm{~mm}$ in the least sensitive range of measurement. Errors in gap width, and in laser scanning of plate height measurement and alignment were neglected. The combined sources of contribution to uncer- 
tainty, through calibration and measurement, in distance to gap and lift-off are given in Table 1, together with their proportion of influence on the result for the case of high influence of drift.

\section{Results}

The complex impedance results can be conveniently visualised as response grids, where each simulation point, or measurement value, is connected to neighbours with the same lift-off and with the same distance, to form a grid of polygons. These grids are rotated and normalized into (arbitrary unit) transformed-X and transformed-Y axes, so that the result of lift-off is registered mainly on the horizontal axis from 0 to -1 , and of distance on the vertical axis. This allows to compare the change in linearity and sensitivity, especially to distance, within the measurement range for each coil and orientation. The calibration grids for both coils, see Section 2.2, can be directly compared to the result from the FE-analysis in [13] by normalising them in the same way, see Fig. 9. The densities of the grids are not the same, since there are more experimental measurement points than numerical calculations. The normalised sensitivity to distance can also be compared, see Fig. 10. From the chart it can be seen that the highest sensitivity at low lift-off is found for distances to the gap between close to zero and up to approximately $2.2 \mathrm{~mm}$. This would result in a desired coil separation in the probe of $2.2 \mathrm{~mm}$ and a working probe range of $\pm 1 \mathrm{~mm}$, which is marked in Fig. 10(a). In contrast, the probe used allowed a minimum coil separation of $4.1 \mathrm{~mm}$ with the corresponding working range marked in Fig. 10(b).

The results of the $100 \mathrm{~mm}$ gap measurements are presented as error maps in Fig. 11. The error is calculated as the difference between the values measured by the inductive probe and the values derived from the laser scanner and the known gap width. Each map shows the maximum of the absolute error in the probe working range for all measurements along the gap, based on the 55 scan lines each with about 200 measurements recorded across the working range. Since the gap is diagonal relative the traverse (as seen in Fig. 6), some of the scans fall outside the probe working range, so the range presented is from -0.8 to $+0.8 \mathrm{~mm}$ in position, rather than from -1 to $+1 \mathrm{~mm}$.

\section{Discussion}

This section addresses the experimental evaluation related to some modelling results, and last influence of uncertainty.

\subsection{Discussion on experimental and modelling results}

For the best performance of a measurement system, it is important to know the properties of the probe and the system. In a typical instrument, the range of the input amplifier must be adapted to the magnitude of the response. For all coils and orientations in this investigation, this is largely determined by the sensitivity to lift-off $\mathrm{L}$, which is much greater than to distance $\mathrm{D}$.

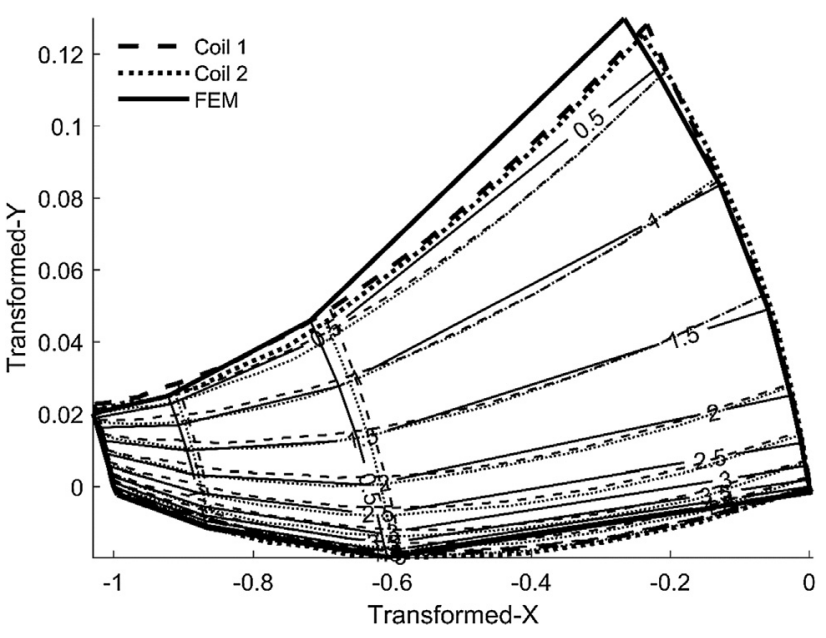

Fig. 9. Normalised response grids for FEM, coil 1 and coil 2 . The outlines of the responses are marked in bold.

With the results from the simulation in [13], the Norm coil was chosen for its higher sensitivity in both absolute and normalised sense. The physical coils were designed as Norm long coils, wound on a $2 \mathrm{~mm}$ diameter core but somewhat longer than in the numerical model. The experimentally measured sensitivity of these coils compare well to the numerically calculated results for this coil design, see Fig. 10. The choice of coil separation can be guided by the sensitivity charts in Fig. 10, which shows that the highest sensitivity at low lift-off is found at a distance $\mathrm{D}$ of $1.1 \mathrm{~mm}$. For higher lift-off L, the best sensitivity has shifted to $\mathrm{D}=1.7 \mathrm{~mm}$. This is closer to the range used in these experiments. Depending on noise, drift and other errors, a trade-off can be made between better accuracy and a larger working range.

It should be noted that the response of the Para coil in [13] could be made stronger by increasing the length of the coil, but that could influence the performance on curved gaps and plates.

The experimental results from the measurement of the $100 \mathrm{~mm}$ long gap, shown as error maps in Fig. 11 underline the importance of sensitivity to distance D. Measurement of position $p$ and, especially of gap width $\mathrm{w}$, shows greater errors than the measurements of height $h$ and alignment $a$. This is also in line with previous results [12] showing the difficulty of compensating errors in measurement on gaps with both gap width and misalignment simultaneously.

\subsection{Discussion on uncertainty}

Some of the sources of uncertainty can have a significant effect on the results as commented in Section 2.4. The instrument drift is the dominating source of uncertainty in both distance and lift-off, and can contribute up to $1 \mathrm{~mm}$ in distance measurement. These large effects would be seen only at the end of measurement runs,

Table 1

Sources of uncertainty and their contribution to measurement in the case of high influence of drift

\begin{tabular}{|c|c|c|c|c|}
\hline \multirow[t]{2}{*}{ Source } & \multicolumn{2}{|c|}{ Distance measurement $d$} & \multicolumn{2}{|c|}{ Lift-off measurement $l$} \\
\hline & Contribution [mm] & Proportion [\%] & Contribution [mm] & Proportion [\%] \\
\hline Electric noise & 0,28 & 7 & 0,06 & 0 \\
\hline Traverse non-lin. & 0,04 & 0 & 0,04 & 4 \\
\hline Electric drift & 1,00 & 92 & 0,20 & 88 \\
\hline Probe height & 0,00 & 0 & 0,02 & 1 \\
\hline Probe angle & 0,00 & 0 & 0,06 & 7 \\
\hline Sum & 1,04 & 100 & 0,22 & 100 \\
\hline
\end{tabular}



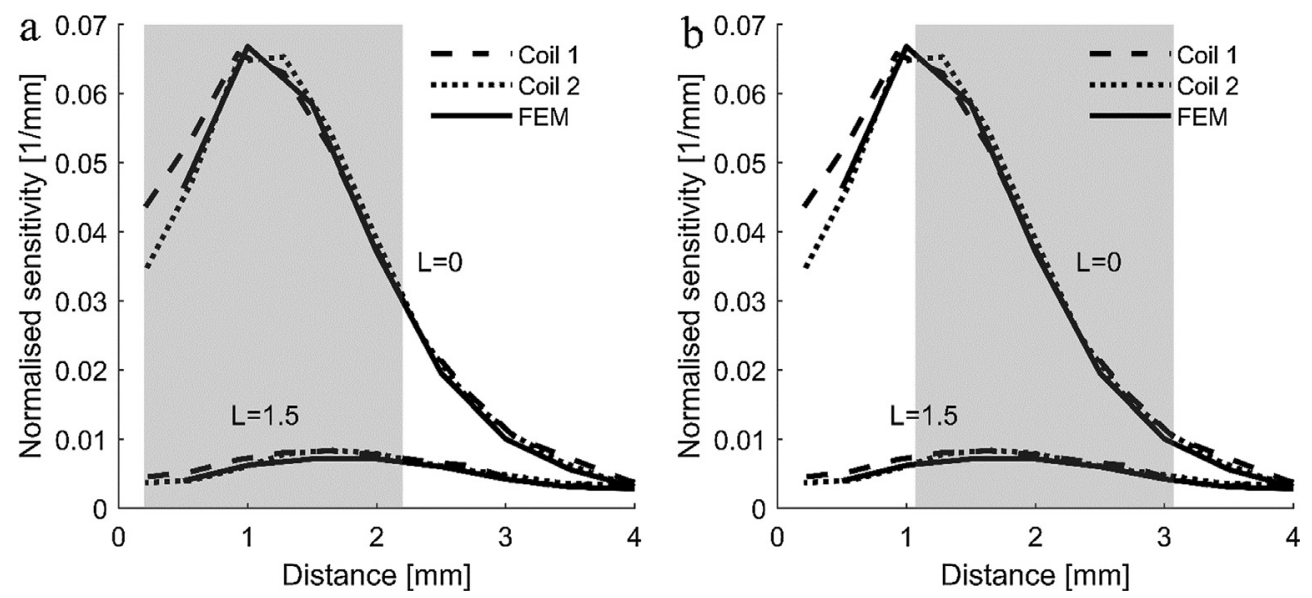

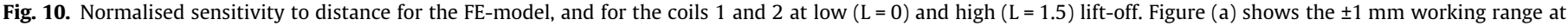
highest sensitivity, figure (b) shows the range used in experiments.
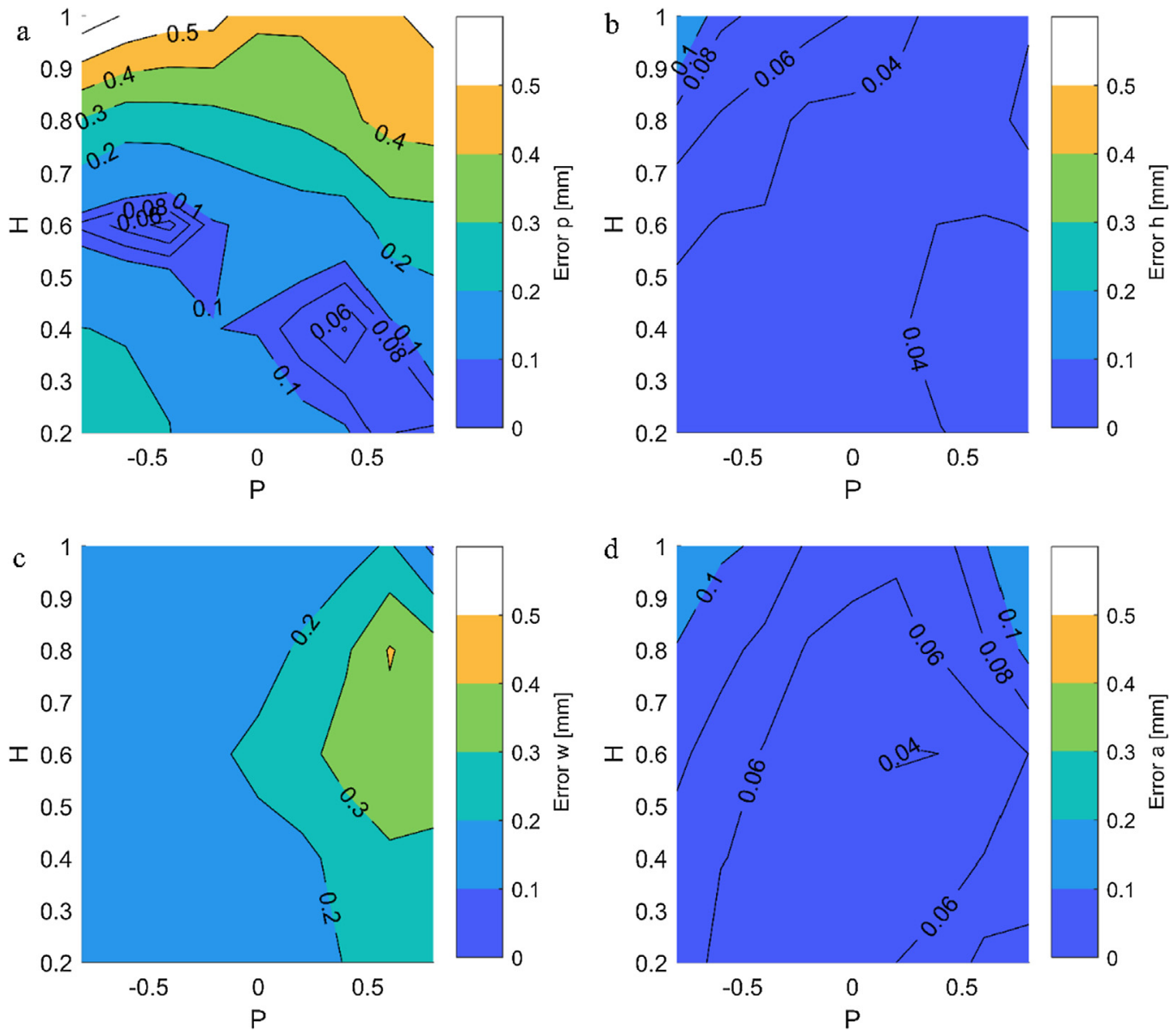

Fig. 11. Errors in (a) position p, (b) height h, (c) gap width w, and (d) alignment a measurements for the working range in $P$ and $H$.

which corresponds to high lift-off values at the upper end of the working range presented in Fig. 11; these measurements were close to $30 \mathrm{~min}$ long. The second largest influence in this part of the working range is the electric noise for distance, and probe angle for lift-off. At lower heights, with higher sensitivity, contributions from the traverse system should also be considered. Manual procedures such as probe centering may also have contributed significantly to the error results. When identified though, the dominating source can be reduced by more frequent instrument zero- ing and more automated procedures. This would normally be the case in industrial use, with shorter weld runs and robotized probe handling. Influence of variation in coil temperature was not considered since compensating coils were used, but differences in coil resistance matching could still cause small effects. Most importantly, the probe should be designed to use the most sensitive part of the coil distance to gap D range. In these experiments, sensitivity to distance $\mathrm{D}$ in the least sensitive part of the working range could have been higher by using a smaller coil separation. Further, as can 
be seen from Fig. 4(a), the sensitivity to distance to gap D for the Norm coil is reduced by increasing the lift-off $\mathrm{L}$ above the plate. For the coils used, the overall sensitivity could have been somewhat larger by reducing the protrusion of the coil core. These are decisions where numerical modelling before starting the design work can be useful.

\section{Conclusion}

The practical experiments verify the numerical results of distance $\mathrm{D}$ sensitivity in relation to lift-off $\mathrm{L}$ response, confirming that numerical modelling can be used to design probes. Further investigation could be directed into for example the influence of changes in gap width and alignment, the effect of curved weld paths and plates, as well as different instrument frequencies.

The measurement of a realistic gap shows results that can be used in industrial applications for position $\mathrm{p}$, plate height $\mathrm{h}$ and height alignment a. Measurement of gap width w needs improvements, such as careful use of the coils' working ranges and instrument drift minimisation by frequent zeroing. Possible improvements to the presented system are identified, most importantly the efficient use of the most sensitive part of the measurement range.

\section{Declaration of Competing Interest}

None.

\section{Acknowledgement}

The authors gratefully acknowledge the financial support from the research school SiCoMaP, funded by the Swedish Knowledge
Foundation (Stiftelsen för kunskaps och kompetensutveckling, KK-Stiftelsen), and GKN Aerospace Engine Systems.

\section{References}

[1] Y.-X. Zhang, S.-W. Han, J. Cheon, S.-J. Na, X.-D. Gao, Effect of joint gap on bead formation in laser butt welding of stainless steel, J. Mater. Process. Technol. 249 (2017) 274-284

[2] B. Regaard, S. Kaierle, R. Poprawe, Seam-tracking for high precision lase welding applications-methods, restrictions and enhanced concepts, J. Laser Appl. 21 (2009) 1.

[3] E. Svenman, A. Runnemalm, A complex response inductive method for improved gap measurement in laser welding, Int. J. Adv. Manuf. Technol. (2016) 1-10

[4] J. J. Henry, Eddy-current seam tracker for electron-beam welders, 1973, Y-1884 United States 10.2172/4429600 Dep. NTIS TIC English.

[5] W.P. Gu, Z.Y. Xiong, W. Wan, Autonomous seam acquisition and tracking system for multi-pass welding based on vision sensor, Int. J. Adv. Manuf. Technol. 69 (2013) 451-460.

[6] K. Haug, G. Pritschow, Robust laser-stripe sensor for automated weld-seamtracking in the shipbuilding industry, in: Industrial Electronics Society, 1998. IECON'98. Proceedings of the 24th Annual Conference of the IEEE, 1998, pp. $1236-1241$.

[7] A.F. Villán, R.G. Acevedo, E.A. Alvarez, A.C. López, D.F. García, R.U. Fernández, et al., Low-cost system for weld tracking based on artificial vision, IEEE Trans. Ind. Appl. 47 (2011) 1159-1167.

[8] P.J. Wang, W.J. Shao, S.H. Gong, P.J. Jia, G. Li, High-precision measurement of weld seam based on narrow depth of field lens in laser welding, Sci. Technol. Weld. Joining 21 (2016) 267-274.

[9] J. Zeng, B. Chang, D. Du, Y. Hong, S. Chang, Y. Zou, A precise visual method for narrow butt detection in specular reflection workpiece welding, Sensors 16 (2016) 1480

[10] J. Fan, F. Jing, L. Yang, T. Long, M. Tan, A precise seam tracking method for narrow butt seams based on structured light vision sensor, Opt. Laser Technol. 109 (2019) 616-626.

[11] J. García-Martín, J. Gómez-Gil, E. Vázquez-Sánchez, Non-destructive techniques based on eddy current testing, Sensors 11 (2011) 2525-2565.

[12] E. Svenman, A. Runnemalm, Model based compensation of systematic errors in an inductive gap measurement method, Measurement 105 (2017) 17-24.

[13] E. Svenman, Modeling of inductive coil geometry for gap position measurement, Procedia Manuf. 25 (2018) 103-110. 